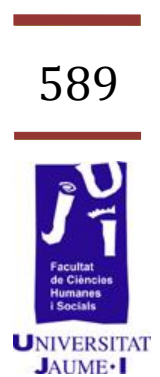

\title{
El evento como instrumento de comunicación eficaz en la gestión del Dircom
}

Mónica Vicente Miravet monica.vte.m@gmail.com 
I. Resumen

El evento se erige como una herramienta de comunicación relacional integrada dentro de la disciplina de las relaciones públicas, que si es utilizada correcta y estratégicamente, puede generar una imagen positiva de una organización en la mente de sus stakeholders, proyectando imagen de marca y desmarcando el producto de los competidores a través de la generación de un valor añadido y diferencial.

La investigación que se va a realizar parte de la hipótesis que inclina a pensar que en ocasiones, el evento es llevado a cabo sin tener perfectamente definidos los objetivos de su celebración. Así, utilizándolo de forma frívola y poco profesional, no se le permite desarrollar su verdadero potencial, que debe basarse en consolidar valores alrededor de la marca, articulándose sobre los objetivos corporativos definidos que se desean cumplir. Cabe resaltar que frecuentemente, los resultados negativos producidos mediante el evento se deben a la falta de profesionalidad en su realización y a su incorrecta gestión en el marco de la estrategia global de la organización; terminando por vincularse a cuestiones meramente lúdicas que no siguen unos objetivos corporativos.

Por lo tanto, se busca verificar científicamente la capacidad del evento de mejorar de forma notable la reputación e imagen positiva que los públicos tienen de una organización. Precisamente, se pretende comprobar que las empresas que utilizan eficazmente el evento bajo las directrices de la dirección de comunicación, de manera organizada dentro de una estrategia de comunicación integral incluida en el plan de comunicación empresarial y formando parte de su eje vertebrador; obtienen mejores resultados que aquellas que no desarrollan la verdadera potencialidad de esta herramienta. Asimismo, también se desea estudiar el previsible desarrollo que las nuevas tecnologías van a desempeñar en este campo, pues su afianzamiento se está viendo incrementado diariamente.

Palabras clave: Comunicación corporativa, dircom, evento, hibridación, marketing, nuevas tecnologías, profesionalidad, relaciones públicas.

\section{Introducción}

\section{Contextualización histórica de las Relaciones Públicas}

La aproximación al concepto de relaciones públicas resulta una tarea compleja, puesto que a lo largo de la historia, se le han adjudicado un gran número de definiciones que han dificultado la existencia de una visión unitaria en torno a ellas. Por ello, con el objetivo de arrojar un halo de luz a esta indefinición que todavía persiste en la actualidad, se va a 
realizar un primer análisis histórico de esta disciplina, que sirva para contextualizar la investigación que se pretende llevar a cabo.

Estados Unidos fue el país pionero en el desarrollo de las relaciones públicas, así como en su posterior consolidación profesional, bajo la denominación original de public relations. Esta disciplina siempre mantuvo un estrecho vínculo con la historia y evolución en el tiempo de la publicidad. Además, nació como una actividad profesional relacionada con el gremio periodístico, habiendo marcado este hecho la evolución temporal de las mismas (Arceo Vacas, 1999: 62-63).

No obstante, se trataría de un error vincular esta materia interdisciplinar tan solo a la América del siglo XX, puesto que otros países como España cuentan con una tradición propia en materia de relaciones públicas, que quizás ha pasado desapercibida en cierto modo debido a la carencia de base bibliográfica (Rodríguez Salcedo, 2007: 184-185).

El estudio The bled manifesto on Public Relations argumenta que las relaciones públicas no deben ser consideradas como una disciplina puramente importada por la Europa de la posguerra desde los Estados Unidos. De este modo, si se atiende a su evolución natural en un país se puede constatar que, fruto del progreso no lineal de las mismas, se han podido diferenciar determinados rasgos para, posteriormente, visionar otros distintos. Y ello conmina a pensar que esta disciplina pudo existir en diversos países europeos con anterioridad a la aludida exportación desde Estados Unidos, habiéndose utilizado diferentes denominaciones para referirse al mismo concepto. Es por ello que el estudio exhaustivo y su correcta contextualización histórica se devienen fundamentales a la hora de plasmar la verdadera identidad de las relaciones públicas (Rodríguez Salcedo, 2007: 185-186).

Se mencionaba anteriormente que la historia de las relaciones públicas está íntimamente ligada a la historia y evolución temporal de la publicidad, no obstante, conviene destacar que pese a ello, estas se desarrollaron como ámbitos separados en Estados Unidos. En cambio, en España sí ha resultado una ardua tarea el poder discernir entre los antecedentes históricos de ambas disciplinas en los orígenes de la actividad, durante la primera mitad del siglo XX. Esta patente incapacidad para definir las funciones básicas de las relaciones públicas ha llevado a realizar estudios -ya en los años sesenta- en los que se muestra cómo campañas de relaciones públicas han permanecido ocultas bajo otras denominaciones tales como publicidad social o publicidad de prestigio. Ello ha llevado a la concepción por parte del grueso de la sociedad a entender las relaciones públicas como un mero instrumento al servicio de la publicidad y demás disciplinas pertenecientes a las ciencias de la comunicación, promoviéndose una incertidumbre patente en su aplicación profesional. (Rodríguez Salcedo, 2007: 201-202). 
Una vez definida la primera contextualización histórica, cabe atender a la afirmación subrayada por diversos estudios históricos. Así, resulta importante exponer que el término relaciones públicas es utilizado habitualmente de forma incorrecta. Ello emana en muchos casos de la intencionalidad manifiesta de denostar dicha profesión o, simplemente, de un problema básico de entendimiento del propio concepto, derivado de la indeterminación sufrida a lo largo de su evolución histórica (Arceo Vacas, 1999: 56). Cualquiera de estos dos aspectos conlleva un gran número de interferencias y dudas acerca de esta disciplina, que fomenta acusadamente aspectos nocivos en la profesión, como el alto nivel de intrusismo profesional existente en la actualidad.

Con todo, cabe destacar el grado de desconocimiento sobre la verdadera procedencia de las relaciones públicas, debido al cual, estas han ido adquiriendo una serie de denominaciones o funciones diversas que no corresponden en modo alguno a su naturaleza e intencionalidad, dejando de valorar así la identidad propia de las mismas. Pese a ello, cabe acentuar que nos encontramos ante una materia interdisciplinar que se nutre de diversas fuentes científicas y que por lo tanto, requiere de un cierto grado de reciprocidad con ellas. No se pretende lograr una ruptura con las demás ciencias de la comunicación, sino reiterar que las relaciones públicas se instauran como una disciplina transversal con entidad propia, que aunque se nutre continuamente de las demás, constituye un concepto híbrido que en modo alguno debe utilizarse únicamente como un instrumento al servicio de otras ciencias.

José Luis Arceo, en su libro Fundamentos para la Teoría y Técnica de las Relaciones Públicas (1988), realiza un recorrido por la evolución de esta profesión, configurando siete etapas fundamentales de su historia, que son (23-48): 1. La prehistoria, 2. Las primeras prácticas, 3. Los primeros fundamentos teórico-técnicos, 4. Avance teórico-técnico y crecimiento, 5. Madurez teórica, técnica y práctica, 6 . Los nuevos posicionamientos y la expansión desfasada, y 7. Refinamientos versus sofisticación. (Arceo Vacas, 1999: 58).

No obstante, los esfuerzos de este y de muchos otros autores no han resultado del todo fructíferos, puesto que en la actualidad continúa sin entenderse adecuadamente el concepto de relaciones públicas, derivando de ello una serie de problemáticas que deberán tratarse de la forma más extensa posible a lo largo de esta investigación, la cual pretende servir de precedente para las siguientes. Por ello, en primer lugar cabe atender a la definición que la Real Academia Española incorpora a sus páginas, en la vigésima segunda edición de su diccionario, en 2001, sobre el término relaciones públicas. Así, el mentado concepto es definido de la siguiente forma: 
sobre personas, empresas, instituciones, etc., tratando de prestigiarlas y de captar voluntades a su favor.

Sin embargo y pese a que esta es la definición oficial que ofrece la Real Academia Española, me permito plasmar otra interpretación del término que, a mi parecer, precisa perfectamente la esencia de las relaciones públicas, afirmando que estas son:

[...] Un tipo de acciones de comunicación, realizadas por las empresas con el fin de crear o modificar la aceptación que por las mismas tienen sus públicos (Bernad Monferrer, et al, 2008: 349).

De este modo, queda patente que las relaciones públicas se convierten en el canal de relación entre la organización y los stakeholders, sirviendo de enlace entre ambos. Los activos intangibles se erigen vitales a la hora de aportar un valor añadido y diferencial a la marca de la que se trate, buscando establecer un vínculo emocional con sus públicos objetivos a través de la comunicación relacional. Así, las relaciones públicas se conectan a las empresas e instituciones para ejecutar ideas que confieran una mayor aceptación de los stakeholders hacia estas.

Por lo tanto, examinado su potencial estratégico, comunicativo y relacional, se deviene fundamental dotar a la disciplina de las relaciones públicas de un rigor científico que permita respetarla; definiéndola como una ciencia empírica que consta de entidad propia y que merece ser positivamente considerada en el sector de las ciencias de la comunicación.

\section{Introducción al evento corporativo y planteamiento de controversias}

Una vez definida la disciplina de las relaciones públicas y acotada atendiendo a su correcta contextualización histórica, cabe aproximarnos ahora al concepto evento. Este se erige como una herramienta de comunicación relacional integrada dentro de la disciplina de las relaciones públicas, que si es utilizada correcta y estratégicamente, puede generar una imagen positiva de una organización en la mente de sus stakeholders, proyectando imagen de marca y desmarcando el producto de los competidores a través de la generación de un valor añadido y diferencial.

Como se ha señalado anteriormente, el objetivo que se persigue con esta investigación es otorgar a las relaciones públicas y por ende, al evento corporativo, un rigor científico que la respete y le otorgue el lugar que verdaderamente le corresponde en el ámbito de la comunicación. Concretamente, considerando este criterio, el evento deberá ser gestionado desde la Alta Dirección a la que pertenece el director de comunicación. Pero esta cuestión será detallada más adelante. En primer lugar, se debe atender a las controversias planteadas en torno al evento.

A la hora de contextualizar históricamente la evolución de las relaciones públicas, ya se ha podido comprobar la existencia de interferencias o 
distorsiones en su denominación y funciones, que han impedido e impiden el correcto entendimiento y ubicación de esta disciplina. Ciertamente, el evento, como herramienta de comunicación relacional integrada en ella, vive la misma situación de desarraigo. Cabe destacar que frecuentemente, los resultados negativos producidos a través del evento se deben a la falta de profesionalidad en su realización, fomentada por el intrusismo y dispersión profesional que se está viviendo, al no comprenderse bien el propio concepto y al ser gestionado incorrectamente en el marco de la estrategia global de la organización. Debido a estas distorsiones que impiden su correcto entendimiento y ubicación en la disciplina de las relaciones públicas, el evento acaba siendo esgrimido como mero elemento de entretenimiento y ocio, sin desarrollar su verdadero potencial que debe basarse en consolidar valores alrededor de la marca, articulándose sobre los objetivos corporativos que se desean cumplir y que deben hallarse previamente definidos en base a una estrategia holística de comunicación corporativa.

Ciertamente, afirmar que los eventos deben ser gestionados por profesionales resulta evidente, hasta el punto de parecer absurdamente obvio. No obstante, si atendemos a la alarmante situación de intrusismo existente en el sector de la organización de eventos corporativos, complementada con la indefinición e incomprensión del concepto relaciones públicas al cual pertenece el evento como instrumento de comunicación; el escenario no parece tan incuestionable. La proliferación constante de agencias de organización de eventos que son dirigidas por profesionales de otros ámbitos, los cuales no disponen de una formación académica que garantice unos conocimientos mínimos de esta materia, debe hacernos reaccionar. La profesionalidad se deviene fundamental para que los eventos sean llevados a cabo con éxito y en base a unos objetivos corporativos planificados, por lo que el intrusismo no solo no beneficia en modo alguno la actual situación del sector, sino que todavía crea una mayor controversia sobre las funciones y objetivos de las relaciones públicas. Con todo, se acaba mostrando un lado meramente lúdico del evento, siendo denostado y poco respetado al no brindarle el lugar que le corresponde al lado de la Alta Dirección.

En referencia a la citada cuestión, cabe abordar otra problemática a la que se enfrenta el sector de la organización de eventos corporativos, la cual corresponde a su manifiesta inclusión en la disciplina del marketing.

Criticar aquí cómo algunos sectores profesionales, en concreto el marketing, intentan hacer ver ante los dirigentes de empresas e instituciones públicas y políticas que los postulados mercadológicos engloban todas las acciones comunicativas, entre ellas, las de relaciones públicas. Nada más lejos de la realidad (Arceo Vacas, 2007: 57).

La afirmación de este autor ha sido también sustentada por otros estudiosos de las relaciones públicas, no obstante, esta sigue siendo la situación real a la que se enfrenta el sector de la organización de eventos, 
considerándose en muchas ocasiones como una herramienta de marketing más y siendo gestionado por los responsables comerciales de las organizaciones en las que se mantiene esta filosofía.

La marca moderna no pertenece al mundo del comercio, sino al de la comunicación (Semprini, 1995: 19).

Esta afirmación ha sido sostenida igualmente por Sanz de la Tajada, el cual tenía la siguiente opinión sobre las relaciones públicas:

[...] Constituyen una función del management y su dependencia directa debe situarse, por tanto, al nivel de la Dirección General. Lo cual no niega sus aportaciones al servicio del marketing, pero siempre de manera indirecta y por vía de la comunicación institucional de la empresa (Castillo Esparcia, 2010: 56).

Asimismo, también Desantes afirmaba que las relaciones públicas no son marketing ni forman parte de él, pues para este autor la problemática generada alrededor de esta disciplina radica, precisamente, en la utilización de términos imprecisos y ambiguos, que ha forjado la indeterminación especificada anteriormente $y$ que ha dado lugar a diferentes y abundantes teorías sobre lo que estas significan.

De este modo, cabe tener en cuenta que el evento es un instrumento de comunicación integrado dentro de la disciplina de las relaciones públicas y por lo tanto, atiende a objetivos relacionales. No obstante, al tratarse de una acción que no puede actuar de forma aislada, esta debe ubicarse eficazmente dentro del plan anual de comunicación de una organización. Esta afirmación está respaldada en que, si se atiende a los objetivos que persigue el marketing, se demuestra que estos son puramente comerciales, en un deseo de alcanzar beneficios económicos muy importantes para la supervivencia de una organización en el mercado. Sin embargo, un evento no debe realizarse atendiendo a objetivos comerciales o en todo caso, estos deben ser secundarios, puesto que este debe encargarse de transmitir eficazmente los atributos relacionados con una marca, con el fin de generar en ella un valor diferencial que le permita destacar por encima de su competencia.

Por lo tanto y dado que es el director de comunicación quien debe gestionar la comunicación global y holística de una organización, entendiendo por ello la gestión de la identidad y la imagen corporativas; también debe confiársele la correcta gestión integral de los eventos como potentes herramientas insertadas en el plan anual de comunicación de la entidad, pues estos, como integrantes de las relaciones públicas, no deben ser gestionados por el marketing sino por la Alta Dirección a la que pertenece el director de comunicación. La finalidad radica, al fin y al cabo, en proyectar de forma eficaz la identidad organizacional para crear fuertes nexos de unión entre las marcas y sus stakeholders, logrando una imagen positiva en la mente de los públicos que se traduzca, a largo plazo, en una buena reputación corporativa. En palabras de Paul Capriotti: 
No hay nada tan real como una creencia, ya que las cosas no son lo que son, sino lo que las personas creen, perciben o conocen de ellas (Matilla Serrano, 2007: 145).

De este modo, volviendo al análisis de las controversias planteadas alrededor del evento, se comprueba que fruto de la indeterminación a la que se ha visto sometida la disciplina de las relaciones públicas, la realidad de los eventos en España todavía está alejada de planteamientos estratégicos que permitan la transmisión de valores a través de la experiencia. Así, pese a que el evento es una herramienta de comunicación en alza y en constante afianzamiento, cabe afirmar que todavía no es entendida en toda su potencialidad comunicativa. Un hecho sorprendente si se tiene en cuenta que casi la totalidad de organizaciones en España tienen como objetivos principales la mejora de relaciones con la marca, la credibilidad y la cobertura mediática (Galmés Cerezo, 2010: 3). Sin embargo, un gran número de organizaciones todavía siguen actuando mediante una filosofía donde impera el cortoplacismo y la lógica numérica, sin valorar el poder de los activos intangibles a medio y largo plazo. Por ello, el evento dista de situarse en el lugar que le corresponde dentro del marco de la estrategia global de comunicación de una organización, optando frecuentemente por herramientas habituales que les permiten evaluar el retorno de la inversión presupuestaria.

\section{La hibridación del evento online: Una novedosa implementación tecnológica}

Precisamente, dejando de lado la estandarización de los procesos de gestión de eventos corporativos, cabe destacar una novedad que está presente en el día a día de esta profesión: la hibridación del evento a través de la comunicación online. De este modo, la implementación de las nuevas tecnologías en los eventos y la optimización de los recursos innovadores que estas ofrecen, se erigen como factores clave para la consecución del éxito del evento. No obstante, este debe ceñirse siempre a una planificada estrategia de comunicación que persiga alcanzar prospectiva, optimizando de esta forma las posibilidades de conseguir notoriedad y visibilidad mediáticas; permitiendo la participación de un cuantioso número de personas desde cualquier lugar geográfico a través de soluciones virtuales.

Además, cabe valorar de forma reflexiva que las redes sociales han supuesto toda una innovación, ofreciendo interactividad y permitiendo alargar el impacto del evento. Por ello, su correcta utilización ofrece ventajas a la hora de hacer llegar la información de forma bidireccional, interactiva e instantánea, forjándose una comunicación continua entre la organización y sus públicos; pues $<<$ [...] la capacidad socializadora de las redes sociales, implica una acción universalizadora y multiplicadora de los contenidos [...] que puede crear una homogeneización de un público de por sí heterogéneo >> (Peña Acuña, et al, 2019: 18). En la actualidad, se 
deviene fundamental estar presente tanto en online como en offline, gestionando correctamente y en todo momento la información que se ofrece. De este modo, el uso de los social media en la gestión de eventos corporativos aporta una serie de ventajas, como son:

\begin{abstract}
La posibilidad de generar una comunidad que mantiene una conversación en espacios como Facebook y Twitter, la identificación de expertos y prescriptores, realizar networking (en redes sociales profesionales como Linkedln o Xing), crear un debate online antes, durante y después del evento $y$, sobre todo, compartir conocimiento gracias a la filosofía User Generated Content (mediante plataformas como Slideshare o Youtube). El correcto empleo de los social media en el diseño de los eventos favorecerá una gestión automatizada, una eficiente promoción, una mayor participación social y la generación de valor por extensión hacia la organización, asociación o federación empresarial que actúa como emisora del mismo. En consecuencia, los eventos 2.0 representan una ruptura con los canales de comunicación unidireccional, en los que no era posible la interacción con los grupos de interés de las organizaciones, para pasar a un nuevo escenario en el que el evento se diseña desde la premisa de esta nueva filosofía y utiliza, por tanto, canales de distribución multidireccional con la participación activa de los individuos, grupos o colectivos vinculados al evento (Campillo Alhama, et al, 2011: 117).
\end{abstract}

Una vez ha quedado establecida la importancia de optar por la hibridación online en los eventos corporativos, ultimo este apartado introductorio afirmando que se prevé un gran potencial presente y futuro en la realización de eventos mixtos, utilizando para ello los medios convencionales y las herramientas que proporciona la tecnología online. Así, aunque la vertiente presencial resulta importante a la hora de transmitir experiencias, la hibridación del evento online a través de su vertiente virtual se erige como una oportunidad para proyectar eficazmente los mensajes de una organización y generar vínculos de unión con los stakeholders.

\title{
III. Objetivos
}

Con el previo análisis del marco documental, se pretende verificar que la disciplina de las relaciones públicas todavía dista de ser entendida adecuadamente como una materia interdisciplinar, fomentando con ello el intrusismo profesional y las interferencias alrededor de este concepto; buscando esclarecerlas a través de esta investigación.

Por otro lado, mediante las entrevistas con profesionales del sector, se considera necesario comprobar si los eventos actuales son llevados a cabo estratégicamente como acciones de comunicación a través de las cuales la organización desea transmitir mensajes corporativos que les permitan alcanzar los objetivos previamente planificados, o si por el contrario, se continua potenciando el lado meramente lúdico del evento, sin entender su potencial experiencial como instrumento de comunicación relacional; llevándose a cabo como mero entretenimiento. 
Asimismo, se quiere estudiar el previsible desarrollo que las nuevas tecnologías van a desempeñar en el campo de la gestión de eventos, pues su afianzamiento se está viendo incrementado diariamente. Finalmente, destacar que se pretende dotar de valor empírico a la investigación mediante la comprobación o refutación de sus hipótesis para que sirva como referente de futuras investigaciones, puesto que esta temática se encuentra poco explorada académicamente hasta el momento y por ende, la indeterminación conceptual continua haciendo mella en la disciplina de las relaciones públicas y en las herramientas que la integran.

\section{Material y metodología}

De este modo, la presente investigación pretende dar respuesta a una hipótesis principal que queda plasmada de la siguiente manera:

El evento corporativo es una herramienta de comunicación relacional con un gran potencial para mejorar de forma notable la reputación e imagen positiva que los públicos tienen de una organización, siempre que se encuentre gestionado estratégica y profesionalmente por el Director de Comunicación e integrado en el plan anual de comunicación de la organización.

De esta primera hipótesis principal se derivan una serie de subhipótesis que también responden a la intencionalidad de esta investigación. En primer lugar, tal y como se ha venido destacando, el concepto relaciones públicas no es utilizado de forma correcta habitualmente, pues se parte de un problema de entendimiento de la propia disciplina, provocando de esta forma interferencias conceptuales e intrusismo profesional, entre otros factores nocivos. Asimismo, cabe comprobar cómo las organizaciones que tramiten profesionalmente los mensajes proyectados a través del evento siguiendo una misma línea estratégica, verán fomentada su imagen de marca y obtendrán mejores resultados que las empresas que no desarrollen su potencial comunicativo de forma estratégica y gestáltica. Precisamente por ello, se pretende verificar que el evento debe ser gestionado profesionalmente desde la dirección de comunicación de cada organización.

Dado que el objetivo primordial de esta investigación se basa en la comprobación o refutación de las hipótesis planteadas, se va a detallar a continuación en qué va a consistir la metodología que se aplicará. En primer lugar, conviene aclarar que esta investigación pretende servir como base para un posterior Trabajo de Investigación científico que versará sobre esta misma temática. De este modo y dado que su extensión será mucho mayor, también la metodología empleada para ese caso se equiparará en cuanto a volumen de análisis, llevándose a cabo, además, un número más amplio de entrevistas así como el estudio en profundidad de casos concretos que perfilen las hipótesis esbozadas. 
Centrándonos ya en esta primera investigación sobre la materia, cabe resaltar que esta comunicación propone una metodología extensa en lo que se refiere al marco documental. De este modo, el análisis planteado en estas páginas se basa en una labor de documentación previa existente en una bibliografía concreta que ha permitido destacar cuál es la problemática conceptual de las relaciones públicas; un inicio documental que ha servido para certificar cuál es el estado de la cuestión actual.

Por otro lado, se ha procedido a la realización y posterior análisis de ocho entrevistas con expertos profesionales de la comunicación, pertenecientes al mundo de la docencia, de la investigación en torno a la comunicación corporativa y/o relaciones públicas y de la propia actividad profesional basada en la gestión de eventos corporativos. Dichos expertos son los siguientes: Alfredo Arceo Vacas -Doctor en Publicidad y Relaciones Públicas, profesor en la Universidad Complutense de Madrid y consultor de Comunicación Corporativa-; Conchi Campillo Alhama Doctora en Sociología y profesora del Departamento de Comunicación y Psicología Social en la Universidad de Alicante-; Fernando Ramos Fernández -Doctor en Ciencias de la Información y profesor de Derecho de la Información y la Publicidad en la Facultad de Ciencias Sociales y de la Comunicación de la Universidad de Vigo-; Magdalena Mut Camacho Doctora en Comunicación Empresarial e Institucional y profesora de Comunicación Audiovisual y Publicidad en la Universitat Jaume I de Castellón-; María Teresa Benlloch Osuna -Profesora de Comunicación Audiovisual y Publicidad en la Universitat Jaume I de Castellón-; María Teresa Otero Alvarado -Presidenta de la Asociación Universitaria de Relaciones Públicas y Protocolo de Andalucía (ARPPA), presidenta de la Organización Internacional de Protocolo y Ceremonial (OICP), doctora en Ciencias de la Información, y profesora de Comunicación Audiovisual, Publicidad y Literatura en la Universidad de Sevilla-; Roberto Regal Quilez -Director de proyectos en la agencia Roberto Regal Eventos-; y Rosa María Torres Valdés -Doctora en Publicidad y Relaciones Públicas, directora de la Unidad de Responsabilidad Social y Relaciones Públicas en la Universidad de Alicante y profesora del Departamento de Comunicación y Psicología Social en la misma universidad.

Así, con las dosis de información recopiladas a partir del marco documental previo, así como del análisis exhaustivo de las correspondientes entrevistas realizadas, se busca extraer una serie de conclusiones sobre la realidad actual de las relaciones públicas y más concretamente, del sector de la organización de eventos; con el propósito de comprobar las hipótesis planteadas. Cabe destacar que, pese a que el grueso de la investigación lo compone el marco conceptual, la realización de las entrevistas a profesionales ha supuesto un soporte significativo a la hora de reforzar las conclusiones extraidas del previo análisis documental. De este modo, se ha considerado importante su elaboración puesto que al tratarse de expertos comunicólogos de ámbitos distintos, 
han aportado perspectivas y opiniones interesantes a la hora de comprobar las hipótesis propuestas.

Pese a que se darán respuesta a las siguientes cuestiones en el apartado de resultados, cabe mostrar cuáles son las preguntas que se les ha formulado a los entrevistados. Son las siguientes:

1. ¿Considera que la disciplina de las relaciones públicas es entendida actualmente como una ciencia empírica que consta de entidad propia?

2. ¿Encuentra que hay un alto grado de intrusismo en el sector de las relaciones públicas y por lo tanto, en las herramientas de comunicación que la integran?

3. ¿Piensa que el evento corporativo llega a considerarse una herramienta de marketing más? ¿Cree que ello viene dado por las interferencias existentes en torno a dicho concepto, o le parece correcta dicha afirmación?

4. ¿Por qué piensa que el evento es denostado y suele ser recordado por su lado meramente lúdico sin instaurarlo en el lugar que le corresponde dentro del ámbito de la comunicación?

5. ¿Considera que el evento debe ser gestionado por la Alta Dirección a la que pertenece el director de comunicación?

6. ¿Cree que todavía existe una necesidad de comprender el evento como un instrumento de comunicación relacional que puede resultar muy efectivo para una organización siendo esgrimido estratégicamente y en base a unos objetivos corporativos definidos?

7. Dígame el nombre de una empresa que considere que utiliza el evento corporativo para fomentar su imagen de marca y generar un positivo nexo de unión entre ella y sus stakeholders.

8. ¿Qué opina de la hibridación del evento, conjuntando convenientemente la vertiente presencial con la online, en aras de optimizar los recursos ofrecidos por las nuevas tecnologías? ¿Cuál cree que será su potencialidad futura?

Una vez analizado el marco documental que indica cuál es el estado actual de la cuestión, así como realizadas las entrevistas con expertos de las relaciones públicas, de la comunicación corporativa y de la gestión de eventos; se va a proceder a desglosar los resultados alcanzados a través de la metodología científica llevada a cabo, en aras de obtener una serie de conclusiones finales que den respuesta a las cuestiones centrales de las que parte esta investigación.

\section{Resultados}

En primer lugar, por lo que se refiere a la comprensión de las relaciones públicas como una ciencia empírica que consta de entidad propia, cabe resaltar que la práctica totalidad de expertos no duda en afirmar que esta materia interdisciplinar solo es entendida en algunos ámbitos académicos muy especializados, habiendo sido superada por la gestión de la reputación, debido a la sólida teoría que la acompaña; en detrimento de la imprecisión conceptual que las relaciones públicas han sufrido desde los inicios de su actividad. Así, han señalado que esta disciplina ha tenido dos enemigos peligrosos: el abuso del término desde 
muy diversas acepciones y usos, y la falta de profesionalidad de quienes asumían su gestión creyendo que se trata de una actividad que cualquier persona puede desempeñar. De este modo, han sido necesarias la investigación a través de la universidad, así como la dignidad de los profesionales conscientes, para intentar situar a esta rama de la comunicación en la posición relevante que le atañe.

Seguidamente, al ser preguntados por el grado de intrusismo enmarcado en la profesión de las relaciones públicas, las tres cuartas partes de los entrevistados aseveran que existe una intrusión profesional considerable; no obstante, matizan que esta no es mayor que la hallada en otras disciplinas pertenecientes al ámbito de la comunicación, tales como el periodismo o la publicidad. Sin embargo, cabe destacar que la problemática del intrusismo en esta materia radica en dos factores que se complementan: la ausencia en un gran número de ocasiones de una preparación mínima para garantizar unos resultados aceptables, y la dispersión encontrada en cuanto a la coexistencia de variados perfiles profesionales que nada tienen que ver con una disciplina académica. Pese a ello, la propia exigencia de efectividad de las organizaciones son factores a favor de la exclusión del intruso y del reconocimiento del verdadero profesional. Por ello, tal y como afirma una cuarta parte de los profesionales, la labor de las Asociaciones de carácter profesional y/o académico está contribuyendo a paliar el problema del instrusismo.

En tercer lugar y haciendo referencia a la inclusión manifiesta del evento corporativo en la disciplina del marketing, se han encontrado discusiones a la hora de alcanzar un resultado concreto que de respuesta a dicha cuestión. Resulta importante destacar que la mayor parte de los entrevistados están de acuerdo en señalar que el evento es una herramienta de comunicación relacional que no pertenece al marketing, no obstante, matizan que según los objetivos que persiga una organización, la naturaleza del evento podrá atender a objetivos comerciales, siempre de forma secundaria y convenientemente conjuntados con los objetivos relacionales perseguidos. Asimismo, coinciden en afirmar que en la mayoría de los casos, el evento se confunde con una herramienta de marketing más. Pese a ser la opinión mayoritaria de los expertos entrevistados, cabe detenerse en la visión de una de las profesionales que asevera que el evento es neutro y que por lo tanto, puede ser una herramienta al servicio de cualquier disciplina de la comunicación, dependiendo siempre del objetivo que se desee alcanzar.

Posteriormente y fruto de la cuestión anterior, ha resultado necesario incidir en la opinión de los profesionales en lo relativo al uso de forma denostada del evento y a la proyección de su lado meramente lúdico. En este caso, cabe mostrar que cada experto tiene su propia visión. Así, una cuarta parte de los entrevistados sostiene que realmente, el evento no es denostado dado que es una herramienta de comunicación muy eficaz si 
se integra adecuadamente en la estrategia de comunicación de la organización de la que se trate. Además, señalan que, por ejemplo, las juntas generales de accionistas, en tanto que son consideradas eventos corporativos, están correctamente diseñadas en base a unos objetivos muy definidos, por lo que esta herramienta suele ser utilizada de forma coherente. Sin embargo, las tres cuartas partes de los expertos señalan que ciertamente, el evento es denostado actualmente, manifestando diversas razones para ello, como son la no adecuada delimitación de los objetivos ponderables del evento, la tradicional falta de planificación estratégica en su gestión, el abuso llevado a cabo contra esta herramienta y la falta de perspectiva académica para abordarlo convenientemente, la ausencia de profesionalización sólida de los organizadores de eventos, o la proyección social que los eventos lúdicos sí han tenido, en detrimento de otros eventos a los que apenas se les ha hecho mención alguna en los medios de comunicación.

En quinto lugar y resultando una cuestión de suma importancia a la hora de comprobar las hipótesis planteadas, los expertos han sido preguntados acerca de la gestión del evento por parte de la Alta Dirección a la que pertenece el director de comunicación. En este caso, la totalidad de los entrevistados no ha dudado en afirmar que, efectivamente, el evento corporativo debe ir en boga con una línea estratégica de comunicación definida y en base a unos objetivos planificados; todo ello gestionado desde la dirección de comunicación. No obstante, muchos de ellos han matizado que, si bien debe ser ejecutado desde la dirección de comunicación, el evento como herramienta será llevado a cabo por personal del equipo de comunicación y no por el propio dircom, que solo se deberá encargar de supervisarlo y asegurarse que este es gestionado acorde con la estrategia de comunicación diseñada por él.

En sexto lugar, atendiendo a la existencia o no de una necesidad de comprender el evento en toda su potencialidad comunicativa, pudiendo resultar una herramienta de comunicación muy efectiva para una organización que lo esgrima de forma eficaz, cabe resaltar que a excepción de una profesional, la amplia mayoría de expertos consideran que todavía existe esa necesidad de comprensión correcta del evento, resultando vital en todo el proceso de diseño y planificación de los objetivos de comunicación para plasmar la estrategia de la organización. Asimismo, subrayan la importancia de saber protocolarizar de forma adecuada todas las fases de interacción con los stakeholders y consolidar los vínculos forjados, con el fin de gestionar de forma adecuada el capital de generación de conocimiento existente en el evento.

Finalmente, haciendo referencia a la novedad existente en torno a la hibridación de los eventos corporativos, conjuntando convenientemente las vertientes virtual y presencial, cabe destacar que una cuarta parte de los entrevistados se aferra a la importancia de la presencialidad a la hora 
de transmitir experiencias por mediación del evento. De este modo, aseguran que el evento como experiencia solo puede ser percibido a través de la asistencia física, pues es ahí donde radica su verdadero valor y fortaleza; perdiendo toda su efectividad si su vertiente virtual es utilizada en la disciplina de las relaciones públicas. No obstante, las tres cuartas partes de los profesionales entrevistados afirman que es fundamental atender a esta doble funcionalidad, pues el poder alcanzar a determinados grupos de interés supone un avance muy importante para una organización. Resulta fundamental elaborar una estrategia bien definida que integre los social media con el resto de canales de comunicación que se van a utilizar, con el fin de coordinar los esfuerzos online con los offline y trabajar de manera adecuada la identidad corporativa de cualquier organización. Así, se gana en rapidez de acceso a la información y además, permite una bidireccionalidad con los públicos, con el fin de escuchar sus expectativas y atenderlas de forma eficaz, integrándolas en los objetivos corporativos de la organización. Concluyen, por lo tanto, que la hibridación del evento es necesaria para optimizar los recursos y alcanzar a más personas, erigiéndose como la gran tendencia presente y futura en el campo de la organización de eventos corporativos.

\section{Conclusiones}

Una vez mostrados los resultados alcanzados a partir de la metodología científica propuesta para esta investigación, resulta importante exponer de forma reflexiva cuáles son las conclusiones a las que la autora llega, a través del estudio del marco conceptual previo y del posterior análisis de las entrevistas llevadas a cabo a diversos profesionales del sector de la comunicación.

En primer lugar, atendiendo a la hipótesis principal que se planteaba, cabe decir que, efectivamente, el evento corporativo es una herramienta de comunicación relacional con un gran potencial para mejorar la reputación e imagen positiva que los públicos tienen de una organización. No obstante, este debe ser gestionado de forma profesional, puesto que, como se ha verificado, en muchas ocasiones las personas que lo llevan a cabo no disponen del conocimiento académico básico en esta materia para garantizar la correcta utilización del evento como herramienta. Asimismo, se confirma que este debe ser gestionado de forma holística por el director de comunicación, el cual ha de diseñar la estrategia comunicacional a seguir en la organización o institución de la que se trate. Sin embargo y en base a las entrevistas realizadas, sí conviene destacar que el dircom se encargará de supervisar que el evento es ejecutado adecuadamente y en línea con la estrategia corporativa definida, no obstante, se requerirá de personal cualificado del equipo de comunicación dependiente del dircom para poner en práctica el evento. 
En segundo lugar, también se ha comprobado que el concepto relaciones públicas no es utilizado de forma correcta habitualmente, subhipótesis verificada a través de un análisis exhaustivo de su historia que ya lo indicaba, así como con la visión de estudiosos en la materia que han certificado dicha afirmación. La utilización de términos imprecisos y ambiguos para referirse al concepto relaciones públicas ha generado su falta de entendimiento y la generación de una serie de interferencias que han provocado que este, solo sea verdaderamente entendido en ámbitos académicos muy especializados. Asimismo, ello ha desencadenado un alto grado de intrusismo profesional en el sector de las relaciones públicas $y$, concretamente, en el sector de la organización de eventos corporativos, provocando la proliferación de agencias que gestionan eventos de forma lúdica, sin unos objetivos corporativos definidos y sin una formación académica adecuada en la materia en cuestión. De este modo, todos estos desencadenantes vienen dados por la dispersión que se ha hecho de las relaciones públicas desde sus inicios. No obstante, conviene destacar que si bien el grado de intrusismo es alto, los profesionales entrevistados han aseverado que otras disciplinas pertenecientes al ámbito de la comunicación no se encuentran exentas de él; aunque desde organizaciones como la Asociación de Empresas Consultoras en Relaciones Públicas y Comunicación (ADECEC), o la Asociación de Directivos de la Comunicación (Dircom), entre otras, se están llevando a cabo estudios para lograr buenas prácticas profesionales y mejoras futuras en el campo de la comunicación.

Por lo que se refiere a la gestión adecuada del evento corporativo, cabe decir que tanto la bibliografía analizada como la opinión de los expertos señalan que el evento es integrado dentro del marketing y tratado, erróneamente, como un instrumento al servicio de esa disciplina. En este caso, estoy de acuerdo con la práctica totalidad de los profesionales que han aseverado que el evento es una herramienta de comunicación relacional y por ende, debe ser gestionado desde la dirección de comunicación de forma holística. Si se parte de la historia y evolución del evento, ya se comprueba cómo este, en tanto que se erige como una herramienta perteneciente a las relaciones públicas, persigue objetivos relacionales, que no coinciden con los comerciales que desea alcanzar el marketing. No puedo estar de acuerdo en considerar el evento como un instrumento neutro al servicio de cualquier disciplina comunicacional, puesto que los objetivos que persiguen no son los mismos. No obstante, cabe reiterar que las relaciones públicas son una materia interdisciplinar que se nutre de las demás disciplinas y por lo tanto, el evento podrá atender a objetivos comerciales de forma secundaria, y siempre que estos se encuentren convenientemente conjuntados con los objetivos relacionales de la organización en cuestión.

Con todo, queda patente que el evento corporativo es una herramienta de comunicación muy potente y eficaz, siempre que forme parte del eje 
vertebrador del plan de comunicación estratégica de una organización y sea gestionado de forma gestáltica bajo las directrices del dircom. Para ello, resulta necesario comprender toda su potencialidad comunicativa, pues las organizaciones que tramiten profesionalmente los mensajes que desean proyectar a través del evento y sigan una misma línea estratégica, verán fomentada su imagen de marca y obtendrán mejores resultados que las empresas que no lo planifiquen profesionalmente y atendiendo a unos objetivos corporativos prefijados. Esta afirmación se manifiesta en el amplio ámbito de influencia del evento corporativo, pues posibilita la interacción con los stakeholders de una organización, creando nexos de unión entre la marca y los públicos estratégicos; y por ello, a través de la relación continua y de la transmisión de experiencias, se origina un mecanismo de escucha importante para cualquier entidad, que posibilita interactuar con los públicos y analizar sus expectativas a fin de facilitar su cumplimiento. Así, el evento puede generar el logro de los objetivos de comunicación de la forma más eficiente: apelando directamente a las emociones de los públicos y consolidando valores alrededor de la marca; logrando, en definitiva, una buena reputación de la organización.

Finalmente, valorar de forma reflexiva que el uso de las nuevas tecnologías en los eventos, apostando por eventos mixtos, se deviene fundamental para su desarrollo presente y futuro. Se ha convertido en una tendencia en alza y por lo tanto, su desarrollo irá acrecentándose progresivamente. Si bien es cierto que hay profesionales que se resisten a creer en la vertiene virtual como complemento de la presencial, no se debe obviar que las ventajas de optar por eventos mixtos son numerosas. De este modo, entiendo la importancia de la presencialidad a la hora de transmitir experiencias y comparto que precisamente esa, ha sido una de sus mayores fortalezas. No obstante, nos encontramos en un momento actual donde el ritmo de vida de cualquier persona es vertiginoso y donde la inmediatez y actualidad juegan un papel trascendental. Las organizaciones responsables deben atender a esta doble funcionalidad, pues supone un avance importante a la hora de poder alcanzar a un número mucho mayor de personas de cualquier lugar geográfico con un streaming muy asequible, permitiendo, además, alargar el impacto del evento y generar grandes dosis de publicity, notoriedad y visibilidad mediáticas muy beneficiosas para cualquier organización. Asimismo, la bidireccionalidad de la información es continua, erigiéndose como otro mecanismo de escucha de las peticiones de los stakeholders, generando una imagen positiva de la entidad en la mente de los mismos. Concluyo afirmando que la hibridación del evento 2.0 a través de su vertiente virtual se está instituyendo como una de las mejores formas de proyectar el mensaje que desea transmitir una organización, gracias a la rapidez, actualidad e interactividad continuas que permite con los stakeholders de cualquier entidad, erigiéndose de este modo como una tendencia al alza en el presente, que se verá acrecentada en el futuro más próximo. 
Arceo Vacas, A. (1999): Estrategias de Relaciones Públicas. Metodologías. Madrid: ICIE, Instituto de Comunicación Institucional y Empresarial de la Universidad Complutense de Madrid, pp. 56-63.

ARCEO VACAS, J.L. (1990): Fundamentos para la Teoría y Técnica de las Relaciones Públicas. Barcelona: Promociones y Publicaciones Universitarias, pp. 23-48.

Bernad Monferrer, E. y M. Mut Camacho (2012): "Redes digitales y evento tradicional: Caso Festapèdia". Revista de Comunicación Vivat Academia, año XIV (№ Especial), Febrero 2012. Madrid. Universidad Complutense, pp. 1431-1444.

Campillo Alhama, C. y otros (eds.) (2011): "La estrategia de comunicación 2.0 en los eventos empresariales". Revista de Comunicación y Nuevas Tecnologías Icono 14, № 8, Octubre 2011. Madrid. II Congreso Internacional Sociedad Digital, pp. 111-126.

CAPRIOTTI PeRI, P. (2009): Branding Corporativo. Fundamentos para la gestión estratégica de la identidad corporativa. Santiago, Chile: Business School Universidad Mayor.

CAstillo Esparcia, A. (2010): Introducción a las Relaciones Públicas. Málaga: Instituto de Investigación en Relaciones Públicas.

CostA, J. (2004): La imagen de marca. Un fenómeno social. Barcelona: Paidós, pp. 171-174 / pp. 158-162.

COSTA, J. (2006): Imagen corporativa en el siglo XXI. Buenos Aires: La Crujía ediciones, pp. 43-77 / pp. 194-226 / pp. 239-251.

Fernández Escalante, F.M. (1989): Ciencias de la Información y Relaciones Públicas. Buenos Aires: Ediciones Macchi.

FUENTE LAFUENTE, C. (2005): Manual práctico para organización de eventos: Técnicas de organización de actos II. Madrid: Protocolo.

FUETTERER, S. y otros (2011). Mi Comunidad... ¿Me quiere o no me quiere? Social Media y Web 2.0 para directivos, comunicadores y emprendedores. Madrid: Best Relations S.A.

GALmÉs CEREZO, M.T. (2010): La organización de eventos como herramienta de comunicación de marketing. Modelo integrado y experiencial. Tesis doctoral dirigida por Juan Salvador Victoria. Universidad de Málaga, 
Facultad de Ciencias de la Comunicación, Departamento de Publicidad y Comunicación Audiovisual.

GRUNIG, J; y T. HUNT (2000): Dirección de relaciones públicas, Barcelona: Gestión 2000, pp. $26-44$.

Jiménez Morales, M. (2005): El plan estratégico de Gestión de Eventos como herramienta para consolidar la imagen de marca. Universitat de Vic: Eumo Editorial. (Estratègies de gestió i comunicació). pp. 161-194.

Jiménez Morales, M. (2007): Manual de gestió d'esdeveniments. La contrucció de la imatge de marca. Universitat de Vic: Eumo Editorial, pp. 9-23 / pp. 143.

Marston, J.E. (1981): Relaciones Públicas Modernas. México: McGraw-Hill.

Matilla Serrano, K. (2007): Aportaciones para un modelo global de Planificación Estratégica en Relaciones Públicas y Comunicación Integral. Tesis doctoral dirigida por Albert Sáez. Facultad de Comunicación, Blanquerna. Universitat Ramón Llull.

Otero Alvarado, M. T. (2007): Tendencias actuales en las Relaciones Públicas Alicante: Asociación de Investigadores de las Relaciones Públicas.

PeÑA AcUÑA, B. y otros (eds.) (2009): "Nuevas características aportadas por el entorno virtual a la comunicación". Revista de Comunicación SEECI, no 19, Julio 2009. Madrid. Universidad Complutense, pp. 1-21.

Real Academia Española (2001): Diccionario de la Lengua Española. Vigésima segunda edición. Madrid: Espasa Calpe.

Rodríguez Salcedo, N. (2007): La historia como tendencia actual de las Relaciones Públicas, en Asociación de Investigadores en Relaciones Públicas (AIRP): Tendencias Actuales en las Relaciones Públicas, pp. 184204.

RUler, B. VAN y D. VerČIČ, (2002), The Bled Manifesto on Public Relations. Bled: Eslovenia, p. 1.

SEMPRINI, A. (1995): El Marketing de la marca. Una aproximación semiótica. Barcelona: Paidós, p. 19.

Vicente Miravet, M. (2012): "Situación comunicativa de las empresas en Castellón a través de la hibridación de eventos online. Estudio de un caso concreto: Congreso Abierto y Virtual Castelló 2020". Revista Estudios Sobre el Mensaje Periodístico, vol. 18 Núm. Especial noviembre (2012). Madrid. Universidad Complutense, pp. 899-907. 
Villafañe, J. (1999): La gestión profesional de la imagen corporativa. Madrid: Pirámide, pp. 29-36.

XIFRA, J. (2007): Técnicas de las Relaciones Públicas. Barcelona: UOC.

\section{Webgrafía}

Web Asociación de directivos de la Comunicación. [En línea]. <http://www.dircom.org/> [Consulta: 22 de diciembre de 2012].

Web Asociación de empresas Consultoras en Relaciones Públicas $y$ COMUNICACIÓN. [En línea]. <http://www.adecec.com> [Consulta: 22 de diciembre de 2012].

Web Portal de Relaciones Públicas. Relaciones Públicas y Gestión de los Públicos en Eventos. [En línea].

<http://www.rrppnet.com.ar/organizaciondeeventos.htm> [Consulta: 17 de diciembre de 2012].

Web Revista Uciencia. Universidad de Málaga. Eventos. [En línea]. <http://www.uciencia.uma.es/Eventos/> [Consulta: 21 de diciembre de 2012]. 